\title{
Myocardial Infarction and Intracerebral Hemorrhage in a Chinese Population: Relationship with Lipoproteins and Adipokines
}

\author{
Jessica Smith $^{1}$, Zhenjun Liu ${ }^{2}$, Huiling Lu, ${ }^{1,2}$, Daowen Wang ${ }^{2}$, Katherine Cianflone ${ }^{1}$ \\ ${ }^{1}$ Centre de Recherche Institut Universitaire de Cardiologie et de Pneumologie de Québec, Québec, Canada \\ ${ }^{2}$ Tongji Medical Centre, HuaZhong University of Science and Technology, Wuhan, China \\ Email: katherine.cianflone@crhl.ulaval.ca \\ Received June 15, 2010; revised August 23, 2010; accepted September 30, 2010
}

\begin{abstract}
BACKGROUND: Adipokines and inflammatory factors play an important role in disease progression. Two cardiovascular diseases which have important contributions to mortality and morbidity in China are intracerebral hemorrhage (ICH) and myocardial infarction (MI). Acylation stimulating protein has been shown in North American populations to have strong associations with risk factors for MI. Complement C3 (C3) a component of the innate complement immune system is the precursor protein to ASP; C3 has been implicated in the pathogenesis of ICH. OBJECTIVE: In this case-control study we examined the association between BMI, lipoproteins adiponectin, C3 and ASP) in a Chinese population. METHODS AND RESULTS: Three groups of subjects were studied: ICH group $(\mathrm{N}=41)$, MI group $(\mathrm{N}=60)$ and a control group $(\mathrm{N}=44)$. There was no difference in BMI for either ICH or MI compared to controls (Control: $22.3 \pm 0.3 \mathrm{~kg} / \mathrm{m}^{2}$; ICH: $21.3 \pm 0.4$ vs MI: $22.5 \pm 0.2, \mathrm{ICH}$ and MI versus control pNS). The ICH group had lower LDL-C (Control: $3.21 \pm 0.13 \mathrm{mmol} / \mathrm{L}$; ICH: $2.54 \pm 0.13$; MI: $2.99 \pm 0.13$; ICH vs control $\mathrm{p}<0.05$ ), total cholesterol (Control: $5.06 \pm 0.16 \mathrm{mmol} / \mathrm{L} ; \mathrm{ICH}: 4.40 \pm 0.15 ; \mathrm{MI}: 4.51 \pm 0.14 ; \mathrm{ICH}$ and $\mathrm{MI}$ vs control $\mathrm{p}<0.05)$, HDL-C (Control: $1.34 \pm 0.05 \mathrm{mmol} / \mathrm{L}$; ICH: $1.22 \pm 0.06$; MI: $0.95 \pm 0.04$; ICH and MI vs control $\mathrm{p}<0.05$ ), and $\mathrm{C} 3$ (Control: $2.58 \pm 0.21 \mathrm{~g} / \mathrm{L} ; \mathrm{ICH}: 1.85 \pm 0.19$; MI: $2.87 \pm 0.16$; ICH vs control $\mathrm{p}<0.05)$, and higher TG (Control: $1.10 \pm$ $0.07 \mathrm{mmol} / \mathrm{L}$; ICH: $1.77 \pm 0.17$; MI: $1.61 \pm 0.10$, ICH and MI vs control $\mathrm{p}<0.05)$, compared to the controls. The MI group had lower total cholesterol and HDL-C and higher TG and ASP (Control: $33.70 \pm 2.07 \mathrm{nM}$; ICH: $35.10 \pm 2.33$; MI: $41.50 \pm 1.81$; MI vs control $\mathrm{p}<0.05$ ) compared to control. CONCLUSION: Chinese men and women who had an MI displayed elevated ASP unrelated to an increase in the precursor protein, C3. Chinese men and women with ICH had ASP levels similar to controls yet lower $\mathrm{C} 3$ suggesting that $\mathrm{C} 3$, and the regulation of $\mathrm{C} 3$ conversion to ASP may be important in ICH disease pathology.
\end{abstract}

Keywords: Myocardial Infarction, Intracerebral Hemorrhage, Acylation Stimulating Protein, Complement C3, Adiponectin

\section{Introduction}

Hemorrhagic stroke, specifically intracerebral hemorrhage (ICH), has a high rate of morbidity and mortality yet it has not received the same intense research interest as ischemic vascular diseases [1]. In fact, in the past 20 years, little progress has been made to improve disease outcomes [2]. In Europe and North America there is a very low incidence of this type of stroke, however, in China, ICH occurs more frequently [3,4]. Economic and social changes that are taking place in many Asian coun- tries, including China, have also led to increasing incidence of ischemic heart diseases, such as myocardial infarction (MI) [5].

The risk factors for MI are well known in Caucasian populations and include obesity, hypertension, and high apolipoprotein B to apolipoprotein A1 (apoB/apoA1) ratio, as well as other factors [6-8]. While these may be widespread risk factors, as suggested by the INTERHEART study, it is unknown if these same risk factors will apply to a Chinese population [6,7]. Recently, increasing rates of obesity, as well as high rates of smoking 
and increasing incidence of insulin resistance and diabetes have begun to coincide with increasing rates of MI in China [9]. By contrast, Chen et al found that a BMI below 20 was also a risk for factor ischemic heart disease in the Chinese population [2]. Hypertension, which is prevalent in the Chinese population, is another major risk factor for both ICH and MI [10,8]. Taken together, these risk factors may be responsible for the higher rates of cardiovascular diseases (CVD) and the development of CVD at a young age in Chinese individuals [11].

In Caucasian populations, obesity and CVD are associated with alterations in several adipose tissue derived hormones, including acylation stimulating protein (ASP), its precursor complement C3 (C3), and adiponectin [12]. It is believed that these hormones may play roles in mediating some co-morbidities of obesity [12] due to their effects on lipid metabolism. ASP increases the uptake of dietary triglyceride (TG) into adipose tissue for storage; however, it is believed that ASP resistance can develop since higher ASP levels are often associated with delays in TG clearance and the development of dyslipidemia.

$\mathrm{ICH}$ and MI have different etiologies and ICH may not be associated with lipid disorders. However, the precursor protein to ASP, C3, is a key component of the complement innate immune system and has been implicated in $\mathrm{ICH}$ disease progression. In rodent models, $\mathrm{C} 3$ gene expression has been shown to increase immediately after the occurrence of an ICH [13] and mice lacking $\mathrm{C} 3$ are protected from further brain damage after ICH $[14,10]$. In humans who have had an $\mathrm{ICH}$, it was reported that they had elevated C3 concentrations [14]. Whether ASP and adiponectin concentrations will be different in individuals who have had an ICH is unknown. Therefore, our goal was to evaluate the concentrations of the hormones ASP, C3 and adiponectin, as well as blood pressure, anthropometric measurements and blood lipids, in subjects who have had MI and ICH in China.

\section{Materials and Methods}

\subsection{Subjects}

One hundred and forty-five participants were recruited at the Tongji Medical Centre, Tongji Hospital, HuaZhong University of Science and Technology, Wuhan, Hubei, China to participate in a case-control study. The subjects were a subset of men and women who had participated in a multicenter study for the assessment of risk factors of stroke sponsored by the Ministry of Science and Technology of China and were included in this analysis based on the following inclusion criteria: non-diabetic (fasting plasma glucose less than $7.0 \mathrm{mmol} / \mathrm{L}$ or casual plasma glucose less than $11.1 \mathrm{mmol} / \mathrm{L}$ ) and age between 45 and 75. Exclusion criteria for all groups included other types of stroke (cerebral thrombosis, lacunar infarction, transient ischemic attack, subarachnoid hemorrhage, embolic brain infarction, brain tumors and cerebrovascular malformation), and severe systemic diseases (collagenosis, endocrine and metabolic diseases, inflammation, liver, neoplastic or renal diseases). Forty-four patients were classified as controls, 41 as having had ICH and 60 as having had MI. Control participants were in-patients at the hospital with minor illnesses from the departments of ophthalmology, gastroenterology, otorhinolaryngology, and orthopedics and were free of neurological diseases or CVD. Diagnosis of ICH was based on neurological examination including CT scan, MRI or both and subjects were evaluated 1 to 12 months after ICH. Diagnosis of MI (ST-segment elevation myocardial infarction) was based on 12-lead ECG and data was collected 1 month after diagnosis. None of the subjects were taking medication at the time of the data collection that affected lipids or blood pressure. Informed consent was obtained and the study was approved by the Tongji Hospital Ethics Committee.

\subsection{Anthropometric Measurements}

Height and weight were measured using standard methods. Body mass index (BMI) was calculated as weight $(\mathrm{kg})$ divided by height squared $\left(\mathrm{m}^{2}\right)$. Each participant completed a questionnaire providing health and lifestyle information including participation in regular exercise (yes or no), smoking status (non smoker, previous smoker and current smoker), and medical information on previous/current diseases and medications. Two measurements each for systolic (SBP) and diastolic blood pressure (DBP) were taken on two different days. Reported values for SBP and DBP are an average of these two measurements.

\subsection{Lipoproteins}

Venous blood samples were collected after an overnight fast (12 hours) from an antecubital vein. The samples were centrifuged, aliquoted and frozen at $-80 \mathrm{C}$. Plasma samples were analyzed for concentrations of nonesterified fatty acids (NEFA), triglycerides (TG), total cholesterol (TC), high-density lipoprotein cholesterol (HDL-C), apolipoprotein B (apoB), apolipoprotein A1 (apoA1), adiponectin, C3 and ASP. Plasma NEFA was determined by colorimetric enzymatic assay (WAKO Chemicals, Tokyo, Japan) and TG was determined via GPO-PAP method. TC was analyzed by COD-PAP. HDL-C concentration was determined using an enzymatic colori- 
metric assay after precipitation of apoB-containing lipoproteins (BCR, Ai-Weihali, autobiochemical analyser). LDL-C was calculated using the Friedewald formula $[\mathrm{LDL}-\mathrm{C}=\mathrm{TCHOL}-(\mathrm{TG} / 2 \cdot 2)-\mathrm{HDL}-\mathrm{C}]$. ApoB and apoA1 were assessed by automated immunoturbidimetric assays (Tina-quant; Roche Diagnostics, Mannhein, Germany). Inter-coefficient and intra-coefficient of variation $(\mathrm{CV})$ for all parameters (except HDL-C) were $<3 \%$. For $\mathrm{HDL}-\mathrm{C}$, inter-CV and intra-CV were $<5 \%$.

\subsection{Adipokines}

Total adiponectin concentration was measured by enzyme-linked immunosorbent assay (ELISA; B-Bridge International, Phoenix, AZ, USA). Plasma C3 concentration was determined by turbidimetric assay using a polyclonal anti-human antibody specific against C3 (Lin-Fei Co, Shanghai, China) and plasma ASP concentration was measured using a sandwich ELISA method previously described in detail [15]. For adiponectin, complement C3 and ASP assays, intra-assay CVs were $<4 \%$ and inter-assay CVs were $<8 \%$.

\subsection{Statistical Analysis}

All results are displayed as mean \pm SEM (standard error) unless stated otherwise. ANOVA analyses with Bonferroni post-hoc test was used to compare means of continuous variables. Kruskal-Wallis ANOVA on Ranks with Dunn's post-hoc test was used to compare means of ordinal variables and non-normally distributed continuous variables, as indicated. Correlations between parameters were calculated using Pearson correlation coefficient for continuous variables. Adjusted means and effects of multiple variables on a single dependent variable were determined using multiple regression models. Adjusted means were compared using multivariate analysis of variance (MANOVA).

\section{Results}

\subsection{Subject Characteristics \& Lipoprotein Profile}

Subject characteristics and lipoprotein profiles are presented in Table 1. No gender differences were found for the parameters listed in Table 1; therefore, data from men and women were analyzed together. For the remaining data, gender differences, where present, are indicated. The BMI of the ICH group was lower compared to the MI group yet there was no difference for either ICH or MI compared to control. The traditional lipoprotein risk factors of elevated TG and low HDL-C were present in both the $\mathrm{ICH}$ and $\mathrm{MI}$ groups. On the other hand, there was no presence of hypercholesterolemia, and, in fact, both ICH and MI groups had lower TC than controls as well as lower LDL-C in the ICH group compared to the controls and more dense LDL particles estimated by the ratio of LDL-C/apoB. There was no difference in NEFA, apoA1, apoB or the apoB/apoA1 ratio between the three groups.

Table 1. Subject characteristics.

\begin{tabular}{|c|c|c|c|c|}
\hline & Control & $\mathrm{ICH}$ & MI & ANOVA \\
\hline $\mathrm{N}$ & 44 & 41 & 60 & \\
\hline Gender $(\mathrm{M} / \mathrm{F})$ & $21 / 23$ & $22 / 19$ & $38 / 22$ & \\
\hline $\begin{array}{l}\text { Smoking } \\
\text { (non/previous/current) }\end{array}$ & $30 / 10 / 4$ & $22 / 10 / 9$ & $31 / 15 / 14$ & \\
\hline Age (years) $\dagger$ & $63.5 \pm 0.76$ & $60.2 \pm 1.41$ & $63.8 \pm 0.82$ & 0.24 \\
\hline BMI kg/m2 & $22.3 \pm 0.31$ & $21.3 \pm 0.39 \mathrm{a}$ & $22.5 \pm 0.21 \mathrm{a}$ & 0.01 \\
\hline $\mathrm{TC} \mathrm{mmol} / \mathrm{L}$ & $5.06 \pm 0.16 \mathrm{ab}$ & $4.40 \pm 0.15 \mathrm{a}$ & $4.51 \pm 0.14 b$ & 0.008 \\
\hline $\mathrm{TG} \dagger \mathrm{mmol} / \mathrm{L}$ & $1.10 \pm 0.07 \mathrm{ab}$ & $1.77 \pm 0.17 \mathrm{a}$ & $1.61 \pm 0.10 b$ & $<0.001$ \\
\hline $\mathrm{NEFA} \dagger \mathrm{mmol} / \mathrm{L}$ & $0.23 \pm 0.01$ & $0.31 \pm 0.03$ & $0.27 \pm 0.02$ & 0.14 \\
\hline $\mathrm{HDL}-\mathrm{C} \uparrow \mathrm{mmol} / \mathrm{L}$ & $1.34 \pm 0.05 \mathrm{a}$ & $1.22 \pm 0.06 b$ & $0.95 \pm 0.04 \mathrm{ab}$ & $<0.001$ \\
\hline LDL-C mmol/L & $3.21 \pm 0.13 \mathrm{a}$ & $2.54 \pm 0.13 a$ & $2.99 \pm 0.13$ & 0.003 \\
\hline apoA1 g/L & $1.22 \pm 0.03 \mathrm{a}$ & $1.12 \pm 0.04 \mathrm{a}$ & $1.14 \pm 0.03$ & 0.07 \\
\hline$a p o B \dagger g / L$ & $0.77 \pm 0.02$ & $0.75 \pm 0.03$ & $0.79 \pm 0.03$ & 0.71 \\
\hline $\mathrm{B} / \mathrm{A} 1 \uparrow \mathrm{g} / \mathrm{L}$ & $0.70 \pm 0.02$ & $0.66 \pm 0.02$ & $0.64 \pm 0.02$ & 0.41 \\
\hline $\mathrm{LDL}-\mathrm{C} / \mathrm{apoB} \dagger \mathrm{mmol} / \mathrm{g}$ & $2.21 \pm 0.15 \mathrm{a}$ & $3.35 \pm 0.23 \mathrm{a}$ & $3.84 \pm 0.26$ & 0.007 \\
\hline $\mathrm{SBP} \mathrm{mmHg}$ & $135.9 \pm 3.4$ & $147.5 \pm 4.0$ & $146.7 \pm 3.3$ & 0.04 \\
\hline DBP mmHg & $82.9 \pm 1.9 \mathrm{a}$ & $93.9 \pm 2.4 \mathrm{a}$ & $87.5 \pm 1.9$ & 0.002 \\
\hline
\end{tabular}

All values expressed as mean \pm SEM (standard error) except smoking status. Statistical significance assessed by One-way ANOVA with Bonferroni post-hoc test, except for parameters marked with $\dagger$ where Kruskal-Wallis One Way Analysis of Variance on Ranks with Dunn's post-hoc test was used due to non-normal distribution. For each parameter, values marked with the same letter indicate $\mathrm{p}<0.05$ for post-hoc tests. 


\subsection{Hypertension}

Chi squared analysis of prevalence of hypertension (defined as SBP/DBP $\geq 140 / 90 \mathrm{~mm} \mathrm{Hg}$ ) revealed that there was a difference in the prevalence of hypertension, with the ICH having significantly higher incidence than control $(p=0.01)$. SBP was significantly different by oneway ANOVA between the three groups. DBP was also significantly different with the ICH group having a significantly higher DBP than controls, even when corrected for BMI (adjusted means; control $82.6 \pm 1.87$ mmHg; ICH $92.3 \pm 2.40$; MI $86.9 \pm 1.89, \mathrm{p}=0.0003$ MANOVA). Both SBP and DBP correlated with BMI (SBP, $\mathrm{R}=0.21, \mathrm{p}=0.02$; DBP, $\mathrm{R}=0.18, \mathrm{p}=0.04$ ) and TG (SBP, $\mathrm{R}=0.19, \mathrm{p}=0.01$; DBP, $\mathrm{R}=0.26, \mathrm{p}=0.003$ ) in a pooled analysis of data from all groups. In addition, adiponectin correlated with DBP $(R=-0.24, p=0.006)$. In a multivariate model, with data from all groups pooled, including BMI, TG and group, only BMI $(\mathrm{p}=0.02)$ and TG $(p=0.05)$ significantly contributed to predicting $12.0 \%(\mathrm{p}=0.002)$ of the SBP variance; while BMI $(\mathrm{p}=$ $0.007)$, TG $(p=0.03)$, and group $(p=0.004)$ all significantly and independently predicted $17.2 \%$ of DBP $(\mathrm{P}<$ $0.001)$.

\subsection{Adipokines}

There were no differences in adiponectin as shown in Figure 1 (Control, $6.10 \pm 0.61 \mathrm{mg} / \mathrm{mL}$; ICH, $4.81 \pm 0.54$; MI, $6.05 \pm 0.45$; pNS, Kruskal-Wallis One Way ANOVA on Ranks), but $\mathrm{C} 3$ was lower in the ICH group compared to both the control and the MI groups (control, $2.58 \pm 0.21 \mathrm{~g} / \mathrm{L} ; \mathrm{ICH}, 1.85 \pm 0.19 ; \mathrm{MI}, 2.87 \pm 0.16 ; \mathrm{p}<$ 0.001 ANOVA) (Figure 1). When analyzed separately for gender, there was a significant difference in $\mathrm{C} 3$ concentration between men and women with ICH (ICH men $2.42 \pm 0.28 \mathrm{~g} / \mathrm{L}$ vs. ICH women $1.23 \pm 0.17 \mathrm{~g} / \mathrm{L}, \mathrm{p}<0.05$, ANOVA with post-hoc test) and the differences seen between control and ICH and between ICH and MI were solely due to the lower $\mathrm{C} 3$ value in $\mathrm{ICH}$ women and not ICH men (data not shown). However, in the control group, there was no difference in $\mathrm{C} 3$ values between men and women. When data from all groups were pooled C3 significantly correlated with HDL-C $(\mathrm{R}=-0.23 \mathrm{p}=$ $0.006)$ and adiponectin correlated with $\mathrm{TG}(\mathrm{R}=-0.21 \mathrm{p}$ $=0.01)$ and apoA1 $(\mathrm{R}=0.22, \mathrm{p}=0.01)$.

ASP was higher in the MI group compared to the control (control, $33.70 \pm 2.07 \mathrm{nM}$; ICH, $35.10 \pm 2.33 \mathrm{nM}$; MI, $41.50 \pm 1.81 \mathrm{nM} ; \mathrm{p}=0.002$, Kruskal-Wallis One Way ANOVA on Ranks). Exercise frequency moderately, yet significantly, correlated negatively with ASP, even when adjusted for BMI (adjusted R2 $=0.10, p=0.003$ ). In addition, ASP correlated with NEFA $(\mathrm{R}=0.21, \mathrm{p}=0.01)$, TG $(\mathrm{R}=0.22 \mathrm{p}=0.01)$ and HDL-C $(\mathrm{R}=-0.26 \mathrm{p}=$
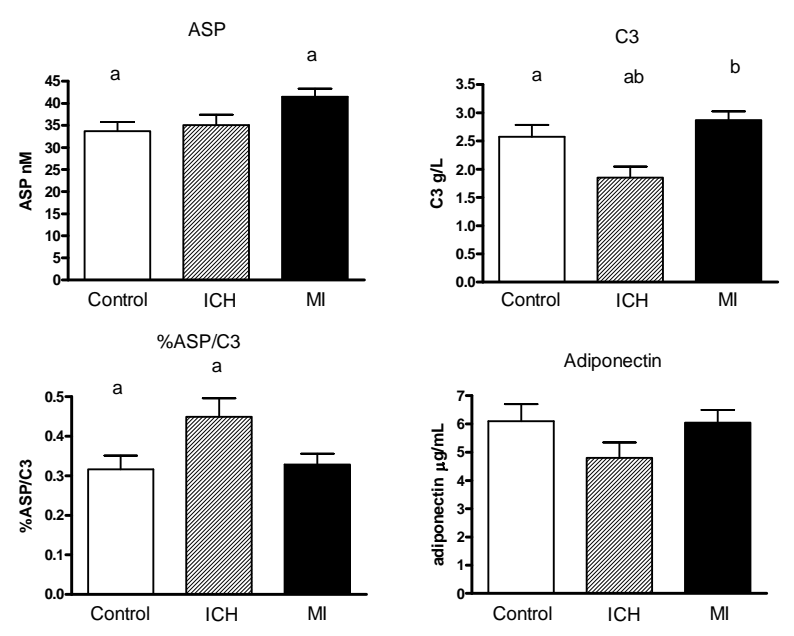

Figure 1. Acylation stimulating protein (ASP, panel A), complement component $\mathrm{C} 3(\mathrm{C} 3$, panel $\mathrm{B})$, the percentage of ASP to C3 (\%ASP/C3, panel C), and adiponectin (panel D) in control, intracerebral hemorrhagic stroke (ICH) and myocardial infarction (MI). Results are presented as mean \pm standard error of the mean (SEM). Statistical significance for $\mathrm{C} 3$ was assessed by one-way ANOVA with Bonferroni post-hoc test. Statistical significance for ASP, \%ASP/C3 and adiponectin assessed by Kruskal-Wallis One Way ANOVA on Ranks with Dunn's post-hoc test due to nonnormal distribution. For each parameter, values marked with the same letter indicate $p<0.05$ for post-hoc tests.

$0.002)$. The $\% \mathrm{ASP} / \mathrm{C} 3$ ratio was significantly lower in the control group versus the ICH group, suggesting that the ICH group had either a higher rate of conversion of $\mathrm{C} 3$ to ASP, or decreased degradation or clearance of ASP (control, $0.32 \pm 0.03 \%$; ICH, $0.45 \pm 0.05 \%$; MI, $0.33 \pm 0.03 \% ; \mathrm{p}=0.005$, Kruskal-Wallis One Way ANOVA on Ranks). Overall, the ICH and MI group had altered lipoprotein profile, altered adipokines and were more hypertensive than controls.

\section{Discussion}

The relationship between blood pressure and MI is well established and is a continuous, positive relationship even at levels below the cut-point for clinical hypertension [8]. There are confounding and additive effects of obesity, hypertension and dyslipidemia on the risk for MI and the independent contribution of each of these factors is difficult to separate. However, in the current study all of the participants were lean and participants within the control and MI groups did not have hypertension. Therefore, dyslipidemia and/or adipokines may potentially be the most prevalent influences for this study.

Several prospective studies on ICH have found a strong positive relationship between ICH and hypertension $[16,17]$ and we found supporting evidence of this: a greater proportion of participants had hypertension in the 
ICH group compared to control or MI. In addition, DBP was higher in the ICH group compared to control, and BMI, TG, and group significantly contributed to predicting DBP. Previous studies have shown that compared to other stroke subtypes, ICH is more strongly associated with hypertension [17], possibly because the small vessels of the brain are vulnerable to damage [18]. The ICH group also had a lower BMI than the other groups and the effects of hypertension may be particularly relevant in the context of low BMI [19].

Hypocholesterolemia has also been suggested as a risk factor for ICH possibly because low cholesterol levels can adversely affect the integrity of the blood vessel in the brain, making them more prone to damage, especially under conditions of hypertension [20,21]. We did find that our ICH group had lower TC and lower LDL-C than the controls. Therefore, our ICH group displayed three potentially exacerbating risk factors for ICH: low BMI, hypocholesterolemia and hypertension. These three factors have not been looked at in combination in predicting the risk of $\mathrm{ICH}$ yet all three are present in our ICH group.

The MI group did not display many of the typical features of atherogenic dyslipidemia seen in Western populations: they had lower TC than controls and had comparable concentrations of NEFA, LDL-C, apoA1, apoB, and apoB/apoA1 to controls. However, the MI group did have elevated TG and low HDL-C. Also, the MI group did not have an elevated BMI, which is typically seen in Western populations with this disease. The factors used to identify 10 year risk of CVD by the Third Report of the National (USA) Cholesterol Education Program Adult Treatment Panel III are age ( $>45$ years for men and $>55$ years considered as high risk), gender (male at higher risk), elevated total cholesterol, low HDL-C, elevated SBP, and smoking status [8]. Much research has also indicated that high apoB and low apoA1 may be are better indicators of risk than cholesterol alone [6]. However, in this Chinese population positive for MI, only a low HDL-C was present. Therefore, many of the Western criteria used to calculate the risk for developing CVD may not be applicable in a lean Chinese population which is nonetheless at risk for developing CVD. Other, non-traditional, risk factors may need to be implemented to correctly identify those at risk, such as adipokines. And, in fact, ASP, which has been linked with CVD in several studies [22], was higher in the MI group compared to controls.

Very few studies have evaluated adipokines in relation to ICH although $\mathrm{C} 3$ has been implicated in ICH pathogenesis $[23,13,10]$. We did see differences in the $\% \mathrm{ASP} / \mathrm{C} 3$, ASP, and $\mathrm{C} 3$ but not adiponectin, as has been shown with ICH and stroke in a previous study [24]. Söderberg and colleagues found no association of adiponectin with hemorrhagic stroke in an European population; however, there were very few cases of hemorrhagic stroke included in their analysis [24].

Complement $\mathrm{C} 3$, as part of the complement immune cascade, has been studied post-ICH. C3-deficient mice are protected from further brain injury due to an inhibition of the immune cascade [25]. In addition, individuals who have had $\mathrm{ICH}$ had increased complement $\mathrm{C} 3$ compared to controls [14], yet in our study individuals who have had an $\mathrm{ICH}$ had lower $\mathrm{C} 3$. While we do not know the concentration of $\mathrm{C} 3$ prior to the brain injury, potentially the lower $\mathrm{C} 3$ could be a survival bias protecting individuals who have had ICH. Our study, along with others, supports further research in the area of C3 in disease prediction, prevention and treatment.

We also found that, despite lower C3, ASP levels were maintained suggesting that there was increased conversion of $\mathrm{C} 3$ to ASP, or a decreased clearance or degradation of ASP in the ICH group. Normally, only a small proportion of $\mathrm{C} 3$ is converted to ASP, and the concentration of $\mathrm{C} 3$ may not be a limiting step in determining the concentration of ASP; however, other mechanisms may be playing a role. It is interesting to note that there were distinct patterns of $\mathrm{C} 3$ and ASP in the MI (normal C3, high ASP) and ICH (low C3, normal ASP) compared to our controls. Thus far, little research has been conducted on the factors that influence the rate of conversion of C3 to ASP, or the factors that influence ASP degradation or clearance. Therefore, further research is need in this area, not only in relation to the pharmacokinetics of $\mathrm{C} 3 / \mathrm{ASP}$ in healthy individuals but how these patterns change in diseases such as ICH and MI. This is the first study to our knowledge which demonstrates different patterns of $\mathrm{C} 3$ and ASP.

This is the first study to our knowledge to measure ASP in an ICH group. In addition, we simultaneously examined several risk factors in both an $\mathrm{MI}$ and $\mathrm{ICH}$ population. Further, understanding the risk factors in a Chinese population, instead of extrapolating data from animal studies or studies from a North American or European population, may improve our ability to prevent and treat these cardiovascular diseases in China.

\section{Acknowledgments}

JS is supported by a CIHR scholarship. KC is the recipient of a senior Canada Research Chair position. This study was supported by grants from CIHR (KC) and National Basic Research Program of China (973 Program, \#2006CB503801 to DWW). 


\section{References}

[1] M. E. Fewel, B. G. Jr Thompson and J. T. Hoff, "Spontaneous Intracerebral Hemorrhage: A Review," Neurosurg Focus, Vol. 15, No. 4, 2003, p. E1.

[2] Z. Chen, G. Yang, M. Zhou, M. Smith, A. Offer, J. Ma, L. Wang, H. Pan, G. Whitlock, R. Collins, S. Niu and R. Peto, "Body Mass Index and Mortality from Ischaemic Heart Disease in A Lean Population: 10 Year Prospective Study of 220,000 Adult Men," International Journal of Epidemiology, Vol. 35, No. 1, 2006, pp. 141-150.

[3] B. Jiang, W. Z. Wang, H. Chen, Z. Hong, Q. D. Yang, S. P. Wu, X. L. Du and Q. J. Bao, "Incidence and Trends of Stroke and Its Subtypes in China: Results from Three Large Cities," Stroke, Vol. 37, No. 1, 2006, pp. 63-68.

[4] L. F. Zhang, J. Yang, Z. Hong, G. G. Yuan, B. F. Zhou, L. C. Zhao, Y. N. Huang, J. Chen and Y. F. Wu, "Proportion of Different Subtypes of Stroke in China," Stroke, Vol. 34, No. 9, 2003, pp. 2091-2096.

[5] L. Wang, L. Kong, F. Wu, Y. Bai and R. Burton, "Preventing Chronic Diseases in China," Lancet, Vol. 366, No. 9499, 2005, pp. 1821-1824.

[6] M. J. McQueen, S. Hawken, X. Wang, S. Ounpuu, A. Sniderman, J. Probstfield, K. Steyn, J. E. Sanderson, M. Hasani, E. Volkova, K. Kazmi and S. Yusuf, "Lipids, Lipoproteins and Apolipoproteins as Risk Markers of Myocardial Infarction in 52 Countries (the INTERHEART study): A Case-Control Study," Lancet, Vol. 372, No. 9634, 2008, pp. 224-233.

[7] X. F. Zhang, J. Attia, C. D'Este, X. H. Yu and X. G. Wu, "A Risk Score Predicted Coronary Heart Disease and Stroke in a Chinese Cohort," Journal of Clinical Epidemiology, Vol. 58, No. 9, 2005, pp. 951-958.

[8] National Cholesterol Education Program (NCEP) Expert Panel on Detection, "Evaluation and Treatment of High Blood Cholesterol in Adults (Adult Treatment Panel III), Third report of the National Cholesterol Education Program (NCEP) Expert Panel on Detection, Evaluation, and Treatment of High Blood Cholesterol in Adults (Adult Treatment Panel III). Final report," Circulation, Vol. 106, No. 25, 2002, pp. 3143-3421.

[9] T. O. Cheng, "The Current State of Cardiology in China," International Journal of Cardiology, Vol. 96, No. 3, 2004, pp. 425-439.

[10] S. Yang, T. Nakamura, Y. Hua, R. F. Keep, J. G. Younger, Y. He, J. T. Hoff and G. Xia, "The Role of Complement $\mathrm{C} 3$ in Intracerebral Hemorrhage-Induced Brain Injury," Journal of Cerebral Blood Flow Metabolism, Vol. 26, No. 12, 2006, pp. 1490-1495.

[11] J. Parry, "China and Japan Face Epidemic of Heart Disease," British Medical Journal, Vol. 329, 2004, p. 643.

[12] M. Faraj, H. L. Lu and K. Cianflone, "Diabetes, Lipids and Adipocytes Secretagogues," Biochemistry Cell Biology, Vol. 82, No. 1, 2004, pp. 170-190.

[13] X. Zhang, H. Li, S. Hu, L. Zhang, C. Lui, C. Zhu, R. Liu and C. Li, "Brain Edema after Intracerebral Hemorrhage in Rats: the Role of Inflammation," Neurology India, Vol. 54, No. 4, 2006, pp. 402-407.
[14] H. F. Chen, X. M. Wang, J. Q. Luo, Z. J. Zheng, R. D. $\mathrm{Wu}$ and $\mathrm{R}$. L. Xu, "Change in Humoral Immunological Function and Their Clinical Significance in Patients with Cerebral Hemorrhage," Chinese Critical Care Medicine, Vol. 17, No. 3, 2005, pp. 177-179.

[15] M. Maslowska, H. Vu, S. Phelis, A. D. Sniderman, B. M. Rhode, D. Blank and K. Cianflone, "Plasma Acylation Stimulating Protein, Adipsin and Lipids in Non-Obese and Obese populations," European Journal of Clinical Investigation, Vol. 29, No. 8, 1999, pp. 679-686.

[16] X. F. Zhang, J. Attia, C. D'Este and X. Y. Ma, "The Relationship Between Higher Blood Pressure and Ischemic, Haemorrhagic Stroke among Chinese and Caucasians: Meta-Analysis," European Journal of Cardio-vascular Prevention Rehabilitation, Vol. 13, No. 3, 2006, pp. 429-437.

[17] H. C. Kim, C. M. Man, S. H. Jee and I. Suh, "Comparison of Blood Pressure - Associated Risk of Intracerebral Hemorrhage and Subarachnoid Hemorrhage: Korea Medical Insurance Corporation Study," Hypertension, Vol. 46, No. 2, 2005, pp. 393-397.

[18] G. R. Sutherland and R. N. Auer, "Primary in-Tracerebral Hemorrhage," Journal of Clinical Neuroscience, Vol. 13, No. 5, 2006, pp. 511- 517.

[19] N. Miyamatsu, T. Kadowaki, T. Okamura, T. Ma-yakawa, Y. Kita, A. Okayama, Y. Nakamura, I. Oki and H. Ueshima, "Different Effects of Blood Pressure on Mortality from Stroke Subtypes Depending on BMI Levels: A 19Year Cohort Study in the Japanese General Population NIPPON DATA80," Journal of Human Hypertension, Vol. 19, 2005, pp. 285-291.

[20] N. Badjatia and J. Rosand, "Intracerebral Hemorrhage," Neurologist, Vol. 11, No. 6, 2005, pp. 311-324.

[21] H. Iso, D. J. Jr Jacobs, D. Wentworth, I. D. Neaton and J. D. Cohen, "Serum Cholesterol Levels and Six Year Mortality from Stroke in 350,977 Men Screened for the Multiple Risk Factor Intervention Trial," The New England Journal of Medicine, Vol. 320, No. 14, 1989, pp. 904-910.

[22] K. Cianflone, Z. Xia and L. Y. Chen, "Critical Review of Acylation-Stimulating Protein Physiology in Humans and Rodents," Biochimica et Biophysica Acta, Vol. 1609, No. 2, 2003, pp. 127-143.

[23] A. F. Ducruet, B. E. Zacharia, Z. L. Hickman, B. T. Gro-belny, M. L. Yeh, S. A. Sosunov and E. S. Jr Connolly, "The Complement Casade as a Therapeutic Target in Intracerebral Hemorrhage," Experimental Neurology, Vol. 219, No. 2, 2009, pp. 398-403.

[24] S. Söderberg, B. Stegmayr, H. Stenlund, L. G. Sjöström, Å. Ågren, L. Johansson, L. Weinehall and T. Olsson, "Leptin, But not Adiponectin, Predicts Stroke in Males," Journal of Internal Medicine, Vol. 256, No. 2,2004, pp. 128-136.

[25] S. Yang, T. Nakamura, Y. Hua, R. F. Keep, J. G. Younger, J. T. Hoff and G. Xi, "Intracerebral Hemorrhage in Complement C3-Deficient Mice," Acta Neurochirurgica Supplement, Vol. 96, 2006, pp. 227-231. 